\title{
Strengths and weakness of neuroscientific investigations of childhood poverty: future directions
}

\author{
Sebastián J. Lipina * and M. Soledad Segretin \\ Unidad de Neurobiología Aplicada (UNA, CEMIC-CONICET), Buenos Aires, Capital Federal, Argentina
}

\author{
Edited by: \\ Daniel S. Margulies, Max Planck \\ Institute for Human Cognitive and \\ Brain Sciences, Germany \\ Reviewed by: \\ Martha Farah, University of \\ Pennsylvania, USA \\ Daniel A. Hackman, University of \\ Pennsylvania, USA \\ *Correspondence: \\ Sebastián J. Lipina, Unidad de \\ Neurobiología Aplicada (UNA, \\ CEMIC-CONICET), Moldes 2152 \\ 1A, Buenos Aires, C1428CRH \\ Capital Federal, Argentina \\ e-mail: lipina@gmail.com
}

The neuroscientific study of child poverty is a topic that has only recently emerged. In comparison with previous reviews (e.g., Hackman and Farah, 2009; Lipina and Colombo, 2009; Hackman et al., 2010; Raizada and Kishiyama, 2010; Lipina and Posner, 2012), our perspective synthesizes findings, and summarizes both conceptual and methodological contributions, as well as challenges that face current neuroscientific approaches to the study of childhood poverty. The aim of this effort is to identify target areas of study that could potentially help build a basic and applied research agenda for the coming years.

Keywords: childhood poverty, social inequality, brain plasticity, interventions, policy making

\section{CURRENT ADVANCES IN THE COMPREHENSION OF BRAIN DEVELOPMENT AND PLASTICITY IN ADVERSE DEVELOPMENTAL CONTEXTS}

The study of how adverse environmental conditions (e.g., socioeconomic status (SES) or poverty) influence brain organization and reorganization during development includes different approaches. Among the most cited factors affecting development are neural plasticity, epigenetics, the influence of environmental toxins, nutrition, stress regulation, poverty modulation of cognitive and emotional processing, cognitive functioning, and health of adults with a history of childhood poverty (Hackman and Farah, 2009; Lipina and Colombo, 2009; Hackman et al., 2010; Bryck and Fisher, 2012; Miller and Chen, 2013). Specifically, current research on the timing of structural and functional development of different neural systems, the multiplicity of levels of organization, and the importance of epigenetics shows that these are important factors in the interpretation of the findings on poverty and brain development. The aim of this section is to highlight the importance of a comprehensive approach to foster the integration of the conceptual models that have been generated in the studies of brain development and plasticity to help design a new generation of research methods and proposals in the study of childhood poverty from a neuroscientific perspective.

The study of the influences of material and social deprivation on the central nervous system (CNS) has been an issue of interest in neuroscience research since the first half of the twentieth century. Early neuroscientific studies in experimental animals analyzed how exposure to complex, standard or deprived environments can modify the brain. At present, the same underlying questions still apply to the analysis of how different rearing environments (i.e., complex vs. standard) modulate brain structure and function at its many different levels (i.e., molecular, genetic, cellular, network, individual, and socialbehavior levels, Hirase and Shinohara, 2014). Specifically, exposure of different species to enriched conditions, in comparison with either standard or deprived environments, has been associated with several structural changes in neurons and synapses, glial components, brain vasculature, brain cortex weight and thickness, rate of hippocampal cell neurogenesis, availability and metabolism of both neurotrophi factors and neurotransmitters in different brain areas, and neurotrophic and neurotransmitter gene expression (Hirase and Shinohara, 2014). It has been proposed that the processes involved in neuroplasticity are affected by different principles (e.g., Mohammed et al., 2002; Hirase and Shinohara, 2014). To this respect, some of the main contributions include the diversity of molecular mechanisms in different brain areas, epigenetic interactions, the role of structural consolidation, inhibitory and excitatory balance, functional competition between inputs, regulation by experience and age, influence of motivation and cognitive control, and potential for reactivation of organizational processes in adulthood (Hensch, 2004; Holtmaat and Svoboda, 2009; Bavelier et al., 2010).

Also, recent behavioral studies have shown that sensitive periods are not necessarily fixed in terms of timing, and suggest that closure of these periods is likely to result from the natural consequence of a given learning process (Michel and Tayler, 2005). In addition, they could coincide with the attainment of functional specialization in a given domain (Johnson, 2005). In the case of the neural circuits involved in complex behaviors, the closure of sensitive periods seems to depend on whether they are associated with circuits performing computations at 
either basic or complex levels, such as feature representation, categorization function, top-down interactions, and cross-modal reorganization (Kral, 2013). Thus, integration of the different forms of plasticity should be the focus for neuroscience research in the field of poverty and brain development aimed at establishing windows for intervention opportunities. This analysis is time-consuming and requires methodological innovations for the exploration of molecular pathways, systems and behavioral events, and phenomena simultaneously, and throughout the different stages of development (e.g., Rao et al., 2010). For instance, in experiments with infants, different tools are usually introduced to facilitate the acquisition of motor skills before the age at which these behaviors are typically observed (Smith and Thelen, 2003). These studies provide behavioral information about how experience-expectant processes can be manipulated to occur earlier than expected in a normal developmental trajectory. Therefore, measurement of neural activity that occurs before the attainment of a certain skill could allow for a better understanding of the development of the mechanisms responsible for these behaviors (e.g., Rao et al., 2010). Another example of the importance of preventivemeasuring of neural activity is the study of how the hearing system is affected differently in contrasting socioeconomic contexts (Skoe et al., 2013). Hearing ability depends on different degrees of environmental noise exposure (Zhou and Merzenich, 2012), and acoustic enrichment of the environment may promote recovery of auditory cortical processing (Zhu et al., 2014).

Similarly to many areas of study on the effects of poverty on development, epigenetic analyses of early brain development in humans are in their early stages. Evidence of the modulation of epigenetic mechanisms during early development in individuals growing under different rearing conditions (e.g., deprived SES, stress exposure) has recently been incorporated into this line of research. For instance, Essex et al. (2013) examined differences in DNA methylation in adolescents for several genes (GR (NR3C1), dopamine receptor (DRD4), serotonin transporter (5HTT), brain-derived neurotrophic factor (BDNF), catechol-Omethyltransferase (COMT), and dopamine transporter (DAT1)) in relation to their parents' reports of hardship during childhood. They found that maternal stress in infancy predicted higher methylation levels in both girls and boys, but paternal stressors in preschool predicted differences in methylation at adolescence specifically in girls. In addition, recent cumulative evidence suggests that differential susceptibility to the rearing environment may depend on variations in dopamine-related genes. For instance, Bakermans-Kranenburg and van Ijzendoorn (2011) found that children with secure attachment representations donated more money to a charity (e.g., UNICEF) in the context of an attachment story completion task, only if they had the DRD4 7-repeat allele; and that children with less efficient dopamine-related genes (D2, DRD4, DAT1) had more adaptive difficulties in negative rearing environments. More recently, these types of molecular genetic approaches are being increasingly used to examine the association between dopaminergic polymorphisms and educational achievement (e.g., Beaver et al., 2012).
Although many conceptual and methodological issues should be explored, initial epigenetic findings support the notion that epigenetic changes underlie, at least partially, the long-term impact of early experiences, and that epigenetic alterations are potentially reversible or modifiable through pharmacological or behavioral intervention (Hensch, 2004). This means that the understanding of the role of the epigenome on the behavioral modifications driven by early experiences could contribute to our understanding of the relationship between childhood poverty and brain development. However, behavioral associations with genotypes in humans should be interpreted with caution because similar experiences may produce different outcomes in different people. These potentially variable outcomes add another level of complexity to the study of how behavior is modulated by early experiences.

\section{STRENGTHS OF THE CURRENT NEUROSCIENTIFIC APPROACH TO STUDY POVERTY}

Some of the main questions currently included in the neuroscientific study of poverty focus on a number of topics already addressed by the fields of developmental psychology, cognitive psychology, and health sciences, especially those regarding the effects and mechanisms of mediation at the behavioral level of analysis (Bradley and Corwyn, 2002; Hackman and Farah, 2009; Moffitt et al., 2011; Evans et al., 2013). The aim of this section is to highlight the contributions made by neuroscientific research, that have allowed the growth and expansion of the field of poverty and brain development in recent years.

One of the areas in which these advancements have been verified is the study of stress regulation in early adverse developmental contexts. For instance, recently, the topic of stress regulation has been included in the study of poverty and cognitive development through different perspectives, such as vulnerability and environmental susceptibility (Ellis and Boyce, 2011; Hackman et al., 2012; Sheridan et al., 2013), the impact of poverty on executive functions (Blair et al., 2011), and even child development policy (Shonkoff and Bales, 2011). In all these approaches, the focus of the analytical efforts was on the analysis of the mechanisms mediating stress responses, which took into consideration a number of guiding principles that could contribute to the understanding of childhood poverty. For example, Ganzel et al. (2010) have suggested that properties (i.e., magnitude, duration and chronicity), and types (e.g., social exclusion vs. physical threat) of stressors in early adverse developmental contexts modulate the impact on neural networks involved in acute and chronic responses to stress. In this regard, future research should investigate the timing and specificity of neural development that is sensitive to stress exposure (Lupien et al., 2009).

In addition, current neuroscientific research in the area of early adverse experience on brain development has begun to incorporate concepts and methodologies derived from advances in epigenetics and the analysis of neural activation in animal and human models. Three sets of problems have started to shape the direction of the research in this area: brain plasticity in prenatal development, reactivity of the amygdala 
to threatening situations, and brain changes associated with adverse life experiences (Gianaros and Manuck, 2010). In concert with these issues, research programs have addressed the influence of malnutrition (Georgieff, 2007) and exposure to different types of pollutants and drugs (Hubbs-Tait et al., 2005) during pre- and post-natal brain development, with significant implications for the neuroscientific study of childhood poverty.

Despite these important advances, the neuroscientific study of human poverty, particularly child poverty, is a topic that has gained attention in the most recent decades. Since the mid-1990s, researchers have applied neurocognitive behavioral paradigms to compare the performance of children with disparate SES, and technological advances in neuroimaging have allowed for the analysis of neural networks (Hackman and Farah, 2009; Lipina and Colombo, 2009; Hackman et al., 2010; Raizada and Kishiyama, 2010; D’Angiulli et al., 2012; Lipina and Posner, 2012; Gianaros and Hackman, 2013). Specifically, tasks involving language, cognitive control and memory demands have provided evidence that suggests that these systems may be the most frequently affected by SES adverse environments.

In addition, a recent topic of interest in neuroscience addresses mechanisms of mediation of childhood poverty on cognitive development (Hackman et al., 2010; Noble et al., 2012; Lipina et al., 2013; Neville et al., 2013a), which allows the identification of potential targets for the design of interventions. In this sense, to generate changes in neurocognitive development, interventions have been introduced recently in the study of attention disorders, dyslexia, dyscalculia, executive functions, and arithmetic performance in samples of children from different SES backgrounds. In all of these studies, there has been an emphasis on the behavioral levels of analysis (e.g., Goldin et al., 2014; Segretin et al., 2014), and both neuroimaging techniques and molecular and behavioral genetics have been included in some cases (Rueda et al., 2005, 2012; Bryck and Fisher, 2012; Espinet et al., 2013; Neville et al., 2013b). All of this should help contribute with the identification and the better comprehension of the mechanisms of mediation of early adversity on brain development. For instance, Brito and Noble (2014) have proposed early linguistic environment and stress as the candidate mechanisms through which poverty influences structural (i.e., language hemisphere, hippocampus, amygdala and prefrontal cortex) and functional (i.e., language, memory, social-emotional processing, cognitive control, self-regulation) brain development, based on recent findings considering different systems and levels of organization. Thus, neuroscientific evidence generated during the last decade in the study of childhood poverty has helped to identify the early linguistic environment and the regulation of stress as two main aspects to consider in dealing with the conceptual and methodological challenges, and as future directions in the area.

In summary, the implementation of the technological advances into the study of how early adversity impacts brain development and plasticity, has allowed neuroscientists to improve the identification of mechanisms of mediation and, consequently, has opened new avenues for the innovation in the design of interventions aimed at fostering the development of different emotional, cognitive and social competences. In such a context, many lines of research that begun their development several decades ago (e.g., stress regulation) are converging in a way that seems to be useful when approaching childhood poverty from contemporary neuroscientific perspectives.

\section{LIMITATIONS, CHALLENGES AND FUTURE DIRECTIONS}

The advances in cognitive neuroscience research have posed several conceptual and methodological challenges in the study of childhood poverty. In terms of impacts, mediating mechanisms, hypotheses and the interpretation of data obtained by applying molecular, behavioral, and neuroimaging techniques seems to focus mainly on the comparison of performance and degree of activation rather than the identification of mediating mechanisms (Hackman and Farah, 2009; Lipina and Colombo, 2009; Hackman et al., 2010; Raizada and Kishiyama, 2010; Lipina and Posner, 2012). In addition, most of the evidence is limited to cross-sectional or short-term longitudinal designs, which present difficulty for understanding changes in the study of brain development in adverse contexts. Moreover, the consideration of sensitive periods for many processes susceptible to different socioeconomic conditions and timing of intervention requires a revision of the agendas in other disciplines addressing childhood poverty (e.g., many disciplines currently contend that the impacts of economic and social deprivation are permanent and irreversible) (D'Angiulli et al., 2012). Thus, incorporating findings that show the time-sensitivity of plasticity into research designs could contribute to revise this way of thinking about human brain development in adverse contexts.

Considering the opportunities and setbacks mentioned in the previous two sections, we propose a set of main points that require reconsideration and optimized approaches. First, we propose to increase the focus on the study of variables, factors and mechanisms that mediate the effects of poverty on different cognitive and emotional processes to complement the analysis of impacts. In this context, it is necessary to take into consideration the structural, electrophysiological, and molecular changes in brain plasticity in terms of (a) how neural operations change after adverse experience; (b) the physiological and biochemical involvement of components related to connectivity between different neural networks; (c) how experience and neuropil transformations contribute to brain functional specialization; and (d) the role of epigenetics, sensitive periods and differential susceptibility in shaping neural networks (Hackman et al., 2010; D’Angiulli et al., 2012; Hirase and Shinohara, 2014).

Second, we propose to deepen the theoretical integration of findings from human and animal models to include the consideration of epigenetic mechanisms, to overcome the limitations of only considering the behavioral or neural levels of analysis (Lipina and Colombo, 2009; Hackman et al., 2010), and to promote the simultaneous analysis of more than one level of organization.

Third, we also propose to expand the theoretical integration across all developmental and cognitive psychology, and to plan 
experiments applying neuroimaging techniques to promote and generate innovative hypotheses and research programs (Crone and Ridderinkhof, 2011; Gianaros and Hackman, 2013). For such a purpose, it is necessary to encourage the design of interventions and the measurement of outcomes driven by theoretical models to include the consideration of underlying mechanisms at different levels of analysis. Additionally, it should be important to use conceptual models aimed at understanding the transfer of gains across different domains beyond laboratory methodologies, such as school and work achievement (Crone and Ridderinkhof, 2011; D'Angiulli et al., 2012; Goldin et al., 2014).

Fourth, we suggest the development of innovative studies directed at analyzing plasticity of complex cognitive and emotional processes, and their respective windows of opportunities for intervention (Lipina and Colombo, 2009; D'Angiulli et al., 2012; Lipina and Posner, 2012). This also implies: (a) the support of methodological innovations in the analysis of neural connectivity for studies that compare different intervention contexts (e.g., home, school, community), its mediators, and the potential requirements for the intervention designs (e.g, Jolles and Crone, 2012; Lipina and Posner, 2012); and (b) the generation of alternative methodologies aimed at overcoming limits in sample size, timing of longitudinal designs, and levels of analysis (Gianaros and Hackman, 2013).

Finally, we find it important to improve the knowledge on the conceptualization of childhood poverty in terms of how children experience deprivation, and the generation of innovative ways to operationalize it in suitable terms for neuroscientific approaches (Lipina et al., 2011). This is especially important since the current neuroscientific evidence on developmental patterns has contributed to our understanding of poverty as a phenomenon much more complex and dynamic than the definitions proposed by other social and human scientific disciplines.

\section{ACKNOWLEDGMENTS}

The authors want to thank CONICET, CEMIC, FONCYT, and Fundación Conectar for their support, their colleagues at the Unidad de Neurobiología Aplicada, and Jennifer Simonds and Lesa Ellis for proofreading the manuscript and making suggestions that contributed to its enrichment.

\section{REFERENCES}

Bakermans-Kranenburg, M. J., and van Ijzendoorn, M. H. (2011). Differential susceptibility to rearing environment depending on dopamine-related genes: new evidence and a meta-analysis. Dev. Psychopathol. 23, 39-52. doi: 10. 1017/s0954579410000635

Bavelier, D., Levi, D. M., Li, R. W., Dan, Y., and Hensch, T. K. (2010). Removing brakes on adult brain plasticity: from molecular to behavioral interventions. J. Neurosci. 30, 14964-14971. doi: 10.1523/JNEUROSCI.4812-10. 2010

Beaver, K. M., Wright, J. P., DeLisi, M., and Vaughn, M. G. (2012). Dopaminergic polymorphisms and educational attainment: results from a longitudinal sample of Americans. Dev. Psychol. 48, 932-938. doi: 10.1037/a0026313

Blair, C., Willoughby, M., Greenberg, M. T., Kivlighan, K. T., Fortunato, C. K., Granger, D. A., et al. (2011). Salivary cortisol mediates effects of poverty and parenting on executive functions in early childhood. Child Dev. 82, 1970-1984. doi: 10.1111/j.1467-8624.2011.01643.x

Bradley, R. H., and Corwyn, R. F. (2002). Socioeconomic status and child development. Annu. Rev. Psychol. 53, 371-399. doi: 10.1146/annurev.psych.53. 100901.135233
Brito, N. H., and Noble, K. (2014). Socioeconomic status and structural brain development. Front. Neurosci. 8:276. doi: 10.3389/fnins.2014.00276

Bryck, R. L., and Fisher, P. A. (2012). Training the brain: practical applications of neural plasticity from the intersection of cognitive neuroscience, developmentalpsychology and prevention science. Am. Psychol. 67, 87-100. doi: $10.1037 / \mathrm{a} 0024657$

Crone, E. A., and Ridderinkhof, K. R. (2011). The developing brain: from theory to neuroimaging and back. Dev. Cogn. Neurosci. 1, 101-109. doi: 10.1016/j.den. 2010.12.001

D’Angiulli, A., Lipina, S. J., and Olesinska, A. (2012). Explicit and implicit issues in the developmental cognitive neuroscience of social inequality. Front. Hum. Neurosci. 6:254. doi: 10.3389/fnhum.2012.00254

Ellis, B. J., and Boyce, W. T. (2011). Differential susceptibility to the environment: toward an understanding of sensitivity to developmental experiences and context. Dev. Psychopathol. 23, 1-5. doi: 10.1017/S095457941000060X

Espinet, S. D., Anderson, J. E., and Zelazo, P. D. (2013). Reflection training improves executive function in preschool-age children: behavioral and neural effects. Dev. Cogn. Neurosci. 4, 3-15. doi: 10.1016/j.dcn.2012.11.009

Essex, M. J., Boyce, W. T., Hertzman, C., Lam, L. L., Armstrong, J. M., Neumann, S. M., et al. (2013). Epigenetic vestiges of early developmental adversity: childhood stress exposure and DNA methylation in adolescence. Child Dev. 84, 58-75. doi: 10.1111/j.1467-8624.2011.01641.x

Evans, G. W., Li, D., and Whipple, S. S. (2013). Cumulative risk and child development. Psychol. Bull. 139, 1342-1396. doi: 10.1037/a0031808

Ganzel, B., Morris, P., and Wethington, E. (2010). Allostasis and the human brain: integrating models of stress from the social and life sciences. Psychol. Rev. 117, 134-174. doi: 10.1037/a0017773

Georgieff, M. K. (2007). Nutrition and the developing brain: nutrient priorities and measurement. Am. J. Clin. Nutr. 85, 614S-620S.

Gianaros, P. J., and Hackman, D. (2013). Contribution of neuroscience to the study of socioeconomic health disparities. Psychosom. Med. 75, 610-615. doi: 10. 1097/psy.0b013e3182a5f9c1

Gianaros, P. J., and Manuck, S. B. (2010). Neurobiological pathways linking socioeconomic position and health. Psychosom. Med. 72, 450-461. doi: 10. 1097/PSY.0b013e3181e1a23c

Goldin, A. P., Hermida, M. J., Shalom, D. E., Elias Costa, M., Lopez-Rosenfeld, M., Segretin, M. S., et al. (2014). Far transfer to language and math of a short software-based gaming intervention. Proc. Natl. Acad. Sci. U S A 111, 6443-6448. doi: 10.1073/pnas.1320217111

Hackman, D. A., Betancourt, L. M., Brodsky, N. L., Hurt, H., and Farah, M. J. (2012). Neighborhood disadvantage and adolescent stress reactivity. Front. Hum. Neurosci. 6:277. doi: 10.3389/fnhum.2012.00277

Hackman, D. A., and Farah, M. J. (2009). Socioeconomic status and the developing brain. Trends Cogn. Sci. 13, 65-73. doi: 10.1016/j.tics.2008.11.003

Hackman, D. A., Farah, M. J., and Meany, M. J. (2010). Socioeconomic status and the brain: mechanistic insights from human and animal research. Nat. Rev. Neurosci. 11, 651-659. doi: 10.1038/nrn2897

Hensch, T. K. (2004). Critical period regulation. Annu. Rev. Neurosci. 27, 549-579. doi: 10.1146/annurev.neuro.27.070203.144327

Hirase, H., and Shinohara, Y. (2014). Transformation of cortical and hippocampal neural circuit by environmental enrichment. Neuroscience 280, 282-298. doi: 10 . 1016/j.neuroscience.2014.09.031

Holtmaat, A., and Svoboda, K. (2009). Experience-dependent structural synaptic plasticity in the mammalian brain. Nat. Rev. Neurosci. 10, 647-658. doi: 10. 1038/nrn2699

Hubbs-Tait, L., Nation, J. R., Krebs, N. F., and Bellinger, D. C. (2005). Neurotoxicants, micronutrients and social environments. Individual and combined effects on children's development. Psychol. Sci. Public Interest 6, $57-$ 121. doi: 10.1111/j.1529-1006.2005.00024.x

Johnson, M. H. (2005). Sensitive periods in functional brain development: problems and prospects. Dev. Psychobiol. 46, 287-292. doi: 10.1002/dev. 20057

Jolles, D. D., and Crone, E. A. (2012). Training developing brain: a neurocognitive perspective. Front. Hum. Neurosci. 6:76. doi: 10.3389/fnhum.2012.00076

Kral, A. (2013). Auditory critical periods: a review from system's perspective. Neuroscience 247, 117-133. doi: 10.1016/j.neuroscience.2013.05.021

Lipina, S. J., and Colombo, J. A. (2009). Poverty and Brain Development during Childhood: An Approach from Cognitive Psychology and Neuroscience. Washington, DC: American Psychological Association. 
Lipina, S. J., and Posner, M. I. (2012). The impact of poverty on the development of brain networks. Front. Hum. Neurosci. 6:238. doi: 10.3389/fnhum.2012. 00238

Lipina, S., Segretin, S., Hermida, J., Prats, L., Fracchia, C., Camelo, J. L., et al. (2013). Linking childhood poverty and cognition: environmental mediators of non-verbal executive control in an Argentine sample. Dev. Sci. 16, 697-707. doi: $10.1111 /$ desc. 12080

Lipina, S. J., Simonds, J., and Segretin, M. S. (2011). Recognizing the child in child poverty. Vulnerable Child. Youth Stud. 6, 8-17. doi: 10.1080/17450128.2010. 521598

Lupien, S. J., McEwen, B. S., Gunnar, M. R., and Heim, C. (2009). Effects of stress throughout the lifespan on the brain, behavior and cognition. Nat. Rev. Neurosci. 10, 434-445. doi: 10.1038/nrn2639

Michel, G. F., and Tayler, A. N. (2005). Critical period: a history of the transition from questions of when, to what, to how. Dev. Psychobiol. 46, 156-162. doi: 10. 1002/dev.20058

Miller, G. E., and Chen, E. (2013). The biological residue of childhood poverty. Child Dev. Perspect. 7, 67-73. doi: 10.1111/cdep.12021

Moffitt, T. E., Arseneault, L., Belsky, D., Dickson, N., Hancox, R. J., Harrington, H. L., et al. (2011). A gradient of childhood self-control predicts health, wealth and public safety. Proc. Natl. Acad. Sci. US A 108, 2693-2698. doi: 10.1073/pnas. 1010076108

Mohammed, A. H., Zhu, S. W., Darmopil, S., Hjerling-Leffler, J., Ernfors, P., Winblad, B., et al. (2002). Environmental enrichment and the brain. Prog. Brain Res. 138, 109-133. doi: 10.1016/S0079-6123(02)38074-9

Neville, H., Stevens, C., Pakulak, E., and Bell, T. A. (2013a). Commentary: neurocognitive consequences of socioeconomic disparities. Dev. Sci. 16, 708712. doi: 10.1111/desc.12081

Neville, H. J., Stevens, C., Pakulak, E., Bell, T. A., Fanning, J., Klein, S., et al. (2013b). Family-based training program improves brain function, cognition and behavior in lower socioeconomic status preschoolers. Proc. Natl. Acad. Sci. U S A 16, 12138-12143. doi: 10.1073/pnas.1304437110

Noble, K. G., Houston, S. M., Kan, E., and Sowell, E. R. (2012). Neural correlates of socioeconomic status in the developing human brain. Dev. Sci. 15, 516-527. doi: 10.1111/j.1467-7687.2012.01147.x

Raizada, R. D. S., and Kishiyama, M. M. (2010). Effects of socioeconomic status on brain development and how cognitive neuroscience may contribute to leveling the playing field. Front. Hum. Neurosci. 4:3. doi: 10.3389/neuro.09.003.2010

Rao, H., Betancourt, L., Giannetta, J. M., Brodsky, N. L., Korczykowski, M., Avants, B. B., et al. (2010). Early parental care is important for hippocampal maturation: evidence from brain morphology in humans. Neuroimage 49, 11441150. doi: 10.1016/j.neuroimage.2009.07.003

Rueda, M. R., Checa, P., and Cómbita, L. M. (2012). Enhanced efficiency of the executive attention network after training in preschool children: immediate changes and effects after two months. Dev. Cogn. Neurosci. 15, S192-S204. doi: 10.1016/j.dcn.2011.09.004

Rueda, M. R., Rothbart, M. K., McCandliss, B. D., Saccamanno, L., and Posner, M. I. (2005). Training, maturation and genetic influences on the development of executive attention. Proc. Natl. Acad. Sci. U S A 102, 14931-14936. doi: 10. 1073/pnas.0506897102

Segretin, M. S., Lipina, S. J., Hermida, M. J., Sheffield, T. D., Nelson, J. M., Espy, K. A., et al. (2014). Predictors of cognitive enhancement after training in preschoolers from diverse socioeconomic backgrounds. Front. Psychol. 5:205. doi: 10.3389/fpsyg.2014.00205

Sheridan, M. A., How, J., Arauio, M., Schamberg, M. A., and Nelson, C. A. (2013). What are the links between maternal social status, hippocampal function and HPA axis function in children? Dev. Sci. 16, 665-675. doi: 10.1111/desc.12087

Shonkoff, J. P., and Bales, S. N. (2011). Science does not speak for itself: translating child development research for the public and its policymakers. Child Dev. 82, 17-32. doi: 10.1111/j.1467-8624.2010.01538.x

Skoe, E., Krizman, J., and Kraus, N. (2013). The impoverished brain: disparities in maternal education affect the neural response to sound. J. Neurosci. 33, 1722117231. doi: 10.1523/JNEUROSCI.2102-13.2013

Smith, L. B., and Thelen, E. (2003). Development as a dynamic system. Trends Cogn. Sci. 7, 343-348. doi: 10.1016/S1364-6613(03)00156-6

Zhou, X., and Merzenich, M. M. (2012). Environmental noise exposure degrades normal listening processes. Nat. Commun. 15:843. doi: 10.1038/ncomms1849

Zhu, X., Wang, F., Hu, H., Sun, X., Kilgard, M. P., Merzenich, M. M., et al. (2014). Environmental acoustic enrichment promotes recovery from developmentally degraded auditory cortical processing. J. Neurosci. 34, 5406-5415. doi: 10. 1523/JNEUROSCI.5310-13.2014

Conflict of Interest Statement: The authors declare that the research was conducted in the absence of any commercial or financial relationships that could be construed as a potential conflict of interest.

Received: 27 December 2013; accepted: 19 January 2015; published online: 11 February 2015.

Citation: Lipina SJ and Segretin MS (2015) Strengths and weakness of neuroscientific investigations of childhood poverty: future directions. Front. Hum. Neurosci. 9:53. doi: 10.3389/fnhum.2015.00053

This article was submitted to the journal Frontiers in Human Neuroscience.

Copyright $(2015$ Lipina and Segretin. This is an open-access article distributed under the terms of the Creative Commons Attribution License (CC BY). The use, distribution and reproduction in other forums is permitted, provided the original author(s) or licensor are credited and that the original publication in this journal is cited, in accordance with accepted academic practice. No use, distribution or reproduction is permitted which does not comply with these terms. 